Cecilia Pompili and Alessandro Brunelli

Division of Thoracic Surgery, Ospedali Riuniti Ancona, Ancona, Italy.

Correspondence: A. Brunelli, Division of Thoracic Surgery, Ospedali Riuniti Ancona, Via Conca 1, 60122, Ancona, Italy. E-mail: brunellialex@gmail.com

Received: Dec 102012 | Accepted after revision: Jan 022013

Conflict of interest: None declared.

\title{
References
}

1 Cykert S, Kissling G, Hansen CJ. Patient preferences regarding possible outcomes of lung resection: what outcomes should preoperative evaluations target? Chest 2000; 117: 1551-1559.

2 Ware JE Jr, Sherbourne CD. The MOS 36-items short form health survey (SF36). I. Conceptual framework and item selection. Med Care 1992; 30: 473-483.

3 Cohen J. Statistical Power Analysis for the Behavioral Sciences. 2nd Edn. Hillsdale, Lawrence Erlbaum Associates, 1988.

4 Schulte T, Schniewind B, Walter J, et al. Age-related impairment of quality of life after lung resection for non-small cell lung cancer. Lung Cancer 2010; 68: 115-120.

5 Burfeind WR Jr, Tong BC, O'Branski E, et al. Quality of life outcomes are equivalent after lobectomy in the elderly. J Thorac Cardiovasc Surg 2008; 136: 597-604.

6 Ferguson MK, Parma CM, Celauro AD, et al. Quality of life and mood in older patients after major lung resection. Ann Thorac Surg 2009; 87: 1007-1012.

7 Brunelli A, Socci L, Refai M, et al. Quality of life before and after major lung resection for lung cancer: a prospective follow-up analysis. Ann Thorac Surg 2007; 84: 410-416.

8 Handy JR Jr, Asaph JW, Skokan L, et al. What happens to patients undergoing lung cancer surgery? Outcomes and quality of life before and after surgery. Chest 2002; 122: 21-30.

9 Jylhä M. What is self-rated health and why does it predict mortality? Towards a unified conceptual model. Soc Sci Med 2009; 69: 307-316.

\section{Inhalational anthrax in a vaccinated soldier}

\author{
To the Editor:
}

The spore-forming bacterium, Bacillus anthracis, primarily affects herbivores but can manifest as cutaneous ( $>95 \%$ of cases), gastrointestinal or pulmonary infection in humans. Pulmonary anthrax, resulting from inhalation of anthrax spores, is rapidly progressive and historically was considered to be invariably fatal. Clinical features include haemorrhagic thoracic lymphadenitis, pulmonary oedema, pleural effusions, leptomeningeal involvement, septic shock and respiratory distress, with death often following within $24 \mathrm{~h}[1,2]$.

Due to the high mortality and potential for use as a biological agent, anthrax vaccines were developed in the 1950s [3]. B. anthracis is composed of three proteins known as protective antigen (PA), oedema factor and lethal factor (LF), with PA named after its ability to provide experimental immunity. Anthrax vaccines demonstrated protection against cutaneous anthrax in tannery workers [3] and vaccination protected rhesus monkeys from inhalational anthrax for up to 2 years [4]. No adequately powered studies exist that demonstrate protection by the vaccine against inhalational anthrax in humans. Despite this, widespread vaccination of military personnel was undertaken by allied forces during the recent Gulf Wars [5].

We present a case of initially unrecognised, inhalational anthrax in a vaccinated member of the armed forces.

A 38-year-old male presented with severe, sudden-onset central chest pain at rest, which was associated with nausea and dizziness. He denied shortness of breath. His past medical history included a "supraclavicular lymph node infection" aged 18 years. He was previously fit and well, apart from an undiagnosed painful right knee sustained in the previous year, which resolved with diclofenac. He had no respiratory problems and no family history of note, was married with one daughter, was a nonsmoker and drank $<4$ units alcohol per week. He was a member of the armed forces and had been deployed on operational tours in the Falklands, Kosovo, Germany, Croatia, Cyprus and, most recently, Iraq 4 years earlier. He had travelled to Disneyland, FL, USA, 2 weeks before admission.

On admission he developed haematemesis and an urgent oesophagogastroduodenoscopy (OGD) demonstrated three chronic gastric ulcers. Whilst recovering from the OGD he developed haemoptysis, fever and respiratory compromise and was admitted to the intensive therapy unit, where he was treated empirically with Tazocin and metronidazole. A chest radiograph demonstrated a markedly widened 


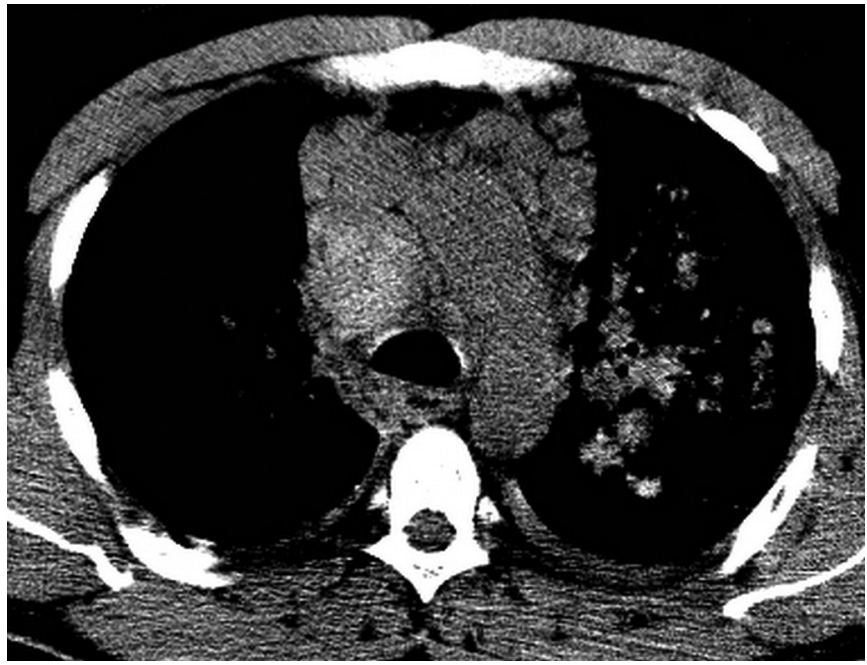

FIGURE 1 Unenhanced computed tomography appearance demonstrating hyperattenuating mediastinal lymph nodes consistent with acute haemorrhagic lymphadenitis.

mediastinum and bilateral pleural effusions. A computed tomography (CT) pulmonary angiogram was negative for thromboembolic disease but demonstrated massively enlarged mediastinal lymph nodes, some of which were hyper-attenuating, bilateral consolidations and pleural effusions. He was anaemic, despite transfusion, with normal urea and electrolytes, liver function tests, clotting and immunoglobulins. Creactive protein (CRP) was elevated at 96 and pneumococcal and Legionella antigens were negative, as was serology for cytomegalovirus and Epstein-Barr virus.

He was transferred to the Royal Brompton Hospital, London, UK where he underwent rigid bronchoscopy and mediastinoscopy with extensive resection of right paratracheal and subcarinal lymph nodes. These showed extensive haemorrhage and necrosis, confirmed on histology. Following the mediastinoscopy his temperature continued to spike at $>38^{\circ} \mathrm{C}$, he developed large bilateral pleural effusions which drained $3 \mathrm{~L}$ of haemorrhagic exudates and remained persistently culture negative from all sites. Further sampling of the subcarinal lymph nodes by endobronchial ultrasound-guided transbronchial needle aspiration confirmed haemorrhagic necrosis and the material obtained was again culture negative. His clinical condition improved with antibiotic treatment, his CRP and temperature settled and treatment was changed to oral co-amoxiclav.

The combination of hyperattenuating (noncalcified) mediastinal lymph nodes on unenhanced CT reflecting a haemorrhagic component, haemorrhagic pleural effusions and histological confirmation of haemorrhagic lymphadenitis suggested the possibility of inhalational anthrax. Further inquiry identified that the patient had been administered anthrax vaccination on four occasions between 2002 and 2003, prior to posting in Iraq, followed by a booster in 2004 .

The Health Protection Agency were contacted for advice. The necrotic mediastinal tissue was examined, confirming that histology was highly suspicious for anthrax. Tissue toxin tests performed were negative. Serum antibody testing identified high PA and LF antibody levels. Acute and convalescent serology demonstrated rising levels of antibodies to both PA and LF antigens.

A retrospective diagnosis of inhalational anthrax was made and the patient was treated for 4 months with co-amoxiclav. Despite exhaustive inquiry, no history of exposure to anthrax spores was identified. Followup radiographs 6 months after presentation demonstrated a normal mediastinal contour.

This is the first case demonstrating survival of inhalational anthrax in a previously vaccinated member of the armed forces and highlights the difficulty associated with the diagnosis of inhalational anthrax in a rapidly deteriorating patient. Our patient had no exposure history and no contact with anthrax was ever identified.

The diagnosis of inhalational anthrax was made in retrospect and would not have been possible had the patient not survived. Despite the suggestive clinical features of haemorrhagic thoracic lymphadenitis and bilateral pleural effusions, which were supported by histology, tissue toxin testing was negative and the diagnosis was made on convalescent antibody levels. The toxin test is sensitive and is positive when anthrax toxin is circulating or present in tissues. It rapidly becomes negative with antibiotic treatment and is cleared from the serum within a day, so would have disappeared by the time the samples in this case were taken.

The histological features of the lymph nodes were highly suspicious of anthrax, and this was substantiated by the extremely high antibody response mounted in this patient. The PA antibody response was at the top of the ELISA scale used to perform the assay, with an optical density double that of recently vaccinated individuals. 
Given the interval of time since the patient's last vaccination, this rising level of PA antibody was unlikely to be due to vaccination alone. High LF antibodies, which are not usually detected in vaccinated individuals, were also detected and acute and convalescent serology demonstrated rising levels of antibodies to both PA and LF antigens. The anti-PA and anti-LF antibodies were measured using a validated ELISA test, which was also employed to study acute cases of anthrax in the concurrent outbreak in drug users. The optical densities of the patient's anti-PA and anti-LF tests were above the upper limit of the linear range of the assay at 3.9 and 3.6, respectively. As the sigmoidal curve of the ELISA (plotted as optical density against dilution of serum) at this point is asymptotic, an exact value cannot be given. This is higher than in any of the 50 positive acute cases of anthrax seen in the same time-frame and considerably higher than the optical densities of 1-2, which fell in the linear range, seen after the initial course of vaccination against anthrax.

The patient developed a serious illness, but it followed a prolonged time-course and he survived. This may have been related to early appropriate empirical antibiotics (Tazocin and metronidazole followed by coamoxiclav) and supportive treatment, but it is also likely that the previous vaccination provided a degree of protection that contributed to his survival. Although historically felt to be universally fatal, survival from inhalational anthrax has improved, especially since the advent of intensive care unit care [6]. Two survivors of bioterrorism-related inhalational anthrax reportedly demonstrated similar features and progress to our patient, including the typical features of mediastinal widening by marked lymphadenopathy (with nodes of increased attenuation due to acute haemorrhage), pleural effusions and air space consolidation [7].

In summary, we have described survival of inhalational anthrax in a previously vaccinated member of the armed forces. The diagnosis was made by a multidisciplinary approach, highlighting the difficulties associated with diagnosing inhalational anthrax and the benefit of multidisciplinary review in complex cases. The patient's survival suggests that previous anthrax vaccination afforded some protection against inhalational anthrax in this case, even several years after vaccination.

Annemarie Sykes ${ }^{1}$, Tim Brooks ${ }^{2}$, Michael Dusmet ${ }^{3}$, Andrew G. Nicholson ${ }^{1,4}$, David M. Hansell ${ }^{5}$ and Robert Wilson ${ }^{6}$ ${ }^{1}$ NHLI Division, Imperial College London, London, ${ }^{2} \mathrm{HPA}$ Centre for Emergency Preparedness and Response, Rare and Imported Pathogens Laboratory, Porton Down, ${ }^{3}$ Dept of Surgery, Royal Brompton and Hospitals NHS Foundation Trust, London, ${ }^{4}$ Dept of Histopathology, Royal Brompton and Hospitals NHS Foundation Trust, London, ${ }^{5}$ Dept of Imaging, Royal Brompton and Hospitals NHS Foundation Trust, London, and ${ }^{6}$ Dept of Respiratory Medicine, Royal Brompton and Hospitals NHS Foundation Trust, London, UK.

Correspondence: A. Sykes, Respiratory Medicine, Imperial College London, Norfolk Place, W2 1PG, UK. E-mail: annemarie.sykes@imperial.ac.uk

Received: Dec 132012 | Accepted after revision: Jan 052013

Conflict of interest: None declared.

\section{References}

1 Abramova FA, Grinberg LM, Yampolskaya OV, et al. Pathology of inhalational anthrax in 42 cases from the Sverdlovsk outbreak of 1979. Proc Natl Acad Sci USA 1993; 90: 2291-2294.

2 Shafazand S, Doyle R, Ruoss S, et al. Inhalational anthrax: epidemiology, diagnosis, and management. Chest 1999; 116: 1369-1376.

3 Brachman PS, Gold H, Plotkin SA, et al. Field evaluation of a human anthrax vaccine. Am J Public Health Nations Health 1962; 52: 632-645.

4 Pile JC, Malone JD, Eitzen EM, et al. Anthrax as a potential biological warfare agent. Arch Intern Med 1998; 158: 429-434.

5 National Network for Immunisation Information. Anthrax. www.immunizationinfo.org/vaccines/anthrax Date last updated: March 11, 2005.

6 Holty JE, Bravata DM, Liu H, et al. Systematic review: a century of inhalational anthrax cases from 1900 to 2005. Ann Intern Med 2006; 144: 270-280.

7 Earls JP, Cerva D Jr, Berman E, et al. Inhalational anthrax after bioterrorism exposure: spectrum of imaging findings in two surviving patients. Radiology 2002; 222: 305-312. 